\title{
ABELIAN MAPS, BI-SKEW BRACES, AND OPPOSITE PAIRS OF HOPF-GALOIS STRUCTURES
}

\begin{abstract}
ALAN KOCH
(Communicated by Martin Liebeck)

Abstract. Let $G$ be a finite nonabelian group, and let $\psi: G \rightarrow G$ be a homomorphism with abelian image. We show how $\psi$ gives rise to two HopfGalois structures on a Galois extension $L / K$ with Galois group (isomorphic to) $G$; one of these structures generalizes the construction given by a "fixed point free abelian endomorphism" introduced by Childs in 2013 . We construct the skew left brace corresponding to each of the two Hopf-Galois structures above. We will show that one of the skew left braces is in fact a bi-skew brace, allowing us to obtain four set-theoretic solutions to the Yang-Baxter equation as well as a pair of Hopf-Galois structures on a (potentially) different finite Galois extension.
\end{abstract}

\section{INTRODUCTION}

Let $G$ be a finite nonabelian group, and let $L / K$ be a Galois extension with Galois group $G$. In 7 Childs introduces the notion of a fixed point free abelian endomorphism of $G$. Given such a map $\psi: G \rightarrow G$ one can endow $L / K$ with a Hopf-Galois structure. Childs furthermore provides a criterion to determine when two different choices of fixed point free abelian endomorphism yield the same HopfGalois structure.

In this work, we introduce a generalization to the above theory. By adjusting how an endomorphism gives rise to a Hopf-Galois structure we are able to improve upon the results in [7] in several meaningful ways. First, we are able to drop the fairly restrictive "fixed point free" condition, thereby obtaining a larger family of HopfGalois structures. Indeed, under Childs's classification, each Hopf-Galois structure emanates from the same Hopf algebra; our generalization allows for more Hopf algebras to act on $L / K$. Second, we simplify the criterion to determine whether endomorphisms give the same Hopf-Galois structure. Finally, in most circumstances (including every case which arises from a fixed point free abelian endomorphism) we are able to find a second Hopf-Galois structure on $L / K$ which is related to the first. While this second structure has been well-known since 1987 (see [10]), Childs's theory lacks an explicit way to describe it; using our modified correspondence this structure becomes transparent.

The past five years have seen a resurgence in Hopf-Galois theory on Galois extensions due to their relationship with set-theoretic solutions to the Yang-Baxter equation. Guarnieri and Vendramin [1] introduced the notion of skew left braces

Received by the editors January 7, 2021, and, in revised form, March 26, 2021.

2020 Mathematics Subject Classification. Primary 16T05, 16T25; Secondary 12F10, 20 N99.

Key words and phrases. Hopf-Galois theory, bi-skew braces, Yang-Baxter equation. 
to provide non-degenerate set-theoretic solutions to this equation, building on the work of Rump, who in [17] developed braces to find solutions that are also involutive. In [1] a connection is given between regular, $G$-stable subgroups of $\operatorname{Perm}(G)$ and left braces for $G$ abelian; this was generalized to the nonabelian case in 19, making the bridge from Hopf-Galois structures and solutions to the Yang-Baxter equation complete: any Hopf-Galois structure on a Galois extension gives rise to such a solution.

Here, given an abelian map $\psi$ we construct what Childs in [5] calls a bi-skew brace. A bi-skew brace is a skew left brace which remains a skew left brace upon interchanging the two binary operations. That the skew left brace is bi-skew has several consequences. First, in the theory of Hopf-Galois extensions a bi-skew brace gives rise to two more Hopf-Galois structures, generally on a Galois extension with a different Galois group (that is, one not isomorphic to $G$ ). Second, a bi-skew brace gives more solutions to the Yang-Baxter equation; using the theory of skew brace opposites as developed independently by Rump in [18 and the author with Truman in 15, a single abelian endomorphism can give up to four different set-theoretic solutions to the Yang-Baxter equation. Two of these solutions appear in [14] in the fixed point free case.

After a quick survey of the basic background material, we introduce abelian maps. Theorem 3.1 establishes the regular, $G$-stable subgroup associated to an abelian map, while corollary 3.2 gives the opposite structure. The connection to 7 ] is given, along with a secondary link to cases where $G$ decomposes as an internal semidirect product as in [8]. The bi-skew brace corresponding to an abelian map is also given, along with (up to) four solutions to the Yang-Baxter equation. We will also find all abelian maps on the symmetric groups $S_{n}, n \geq 5$, the metacyclic groups $M_{p, q}$ of order $p q$ with $p, q$ prime, and all dihedral groups $D_{n}$.

Throughout, $G$ is a finite, nonabelian group with center $Z(G)$, and $L / K$ is a Galois extension with Galois group $G$. We denote by $C_{n}$ the cyclic group of order $n$, written multiplicatively.

\section{BACKGROUND}

Here, we will provide much of the background needed for the subsequent sections.

2.1. Hopf Galois extensions and Greither-Pareigis theory. In [10, Greither and Pareigis develop a powerful theory to find Hopf-Galois structures which we shall briefly outline here-see, e.g., [6] for a detailed treatment.

Let $\operatorname{Perm}(G)$ denote the group of permutations of $G$. For $\eta \in \operatorname{Perm}(G)$ we will denote the image of $g \in G$ under $\eta$ by $\eta[g]$. We say a subgroup $N \leq \operatorname{Perm}(G)$ is regular if for all $g, h \in G$ there is a unique $\eta \in N$ such that $\eta[g]=h$. The simplest examples of such subgroups are the image of $G$ under left regular representation $\lambda: G \rightarrow \operatorname{Perm}(G)$ and right regular representation $\rho: G \rightarrow \operatorname{Perm}(G)$. Notice that these two regular subgroups commute with each other: $\lambda(g) \rho(h)=\rho(h) \lambda(g)$ for all $g, h \in G$.

Clearly, $\lambda(G)$ acts on $\operatorname{Perm}(G)$ via conjugation, i.e. $(\lambda(g), \eta) \mapsto{ }^{g} \eta:=\lambda(g) \eta \lambda\left(g^{-1}\right)$ $\in \operatorname{Perm}(G)$ for $g \in G, \eta \in \operatorname{Perm}(G)$. A subgroup $N \leq \operatorname{Perm}(G)$ is said to be $G$ stable if ${ }^{g} \eta \in N$ for all $g \in G, \eta \in N$. Note that since ${ }^{g} \lambda(h)=\lambda\left(g h g^{-1}\right) \in \lambda(G)$ and ${ }^{g} \rho(h)=\rho(g) \in \rho(G)$ both $\lambda(G)$ and $\rho(G)$ are $G$-stable.

Suppose $N \leq \operatorname{Perm}(G)$ is regular and $G$-stable. By [10, Lemma 2.4.2] the subgroup $N^{\prime}=\operatorname{Cent}_{\operatorname{Perm}(G)}(N)=\left\{\eta^{\prime} \in N^{\prime}: \eta \eta^{\prime}=\eta^{\prime} \eta\right.$ for all $\left.\eta \in N\right\}$ is regular, 
$G$-stable, and is isomorphic to $N$. We will call this the opposite subgroup to $N$, terminology which is justified by the fact that there is a canonical isomorphism $N^{\prime} \rightarrow N^{\text {opp }}$ [10, Lemma 2.4.2]. As a simple example, $\lambda(G)^{\prime}=\rho(G)$.

In 10] a one-to-one correspondence between Hopf-Galois structures on $L / K$ and regular, $G$-stable subgroups is given. For $N \leq \operatorname{Perm}(G)$ the corresponding $K$-Hopf algebra is the fixed ring $L[N]^{G}$, where $g \in G$ acts on $N$ via conjugation via $\lambda(g)$ and on $L$ through the Galois action. Furthermore, we will call the Hopf-Galois structure obtained from $N^{\prime}$ the opposite Hopf-Galois structure to $L[N]^{G}$.

Taking $N=\rho(G)$ gives the usual Galois action. Taking $N=\lambda(G)$ produces what is called the canonical nonclassical Hopf Galois structure in 20]. This structure will be of particular importance here and we shall denote its Hopf algebra by $H_{\lambda}$.

Generally, for $N \leq \operatorname{Perm}(G)$ regular, $G$-stable, the corresponding Hopf-Galois structure with Hopf algebra $H=L[N]^{G}$ is said to be of type $N$. Note that $\lambda(G)$ and $\rho(G)$ are evidently of type $G$.

2.2. Fixed point free abelian endomorphisms. Childs's use of fixed point free abelian endomorphism in [7] provides a useful construction of regular, $G$-stable subgroups. Here, an endomorphism $\psi: G \rightarrow G$ is said to be fixed point free if for every nontrivial $g \in G$ we have $\psi(g) \neq g$; and abelian if $\psi(G) \leq G$ is abelian. For brevity, we will often refer to our endomorphisms as "maps". Note that an abelian map is constant on conjugacy classes. A classification of fixed point free abelian maps on certain classes of finite groups is well understood: see [3, 7, 14.

Given a fixed point free abelian endomorphism $\psi: G \rightarrow G$, we let

$$
N=N_{\psi}=\{\lambda(g) \rho(\psi(g)): g \in G\} .
$$

It is easy to verify that $N$ is a regular, $G$-stable subgroup of $\operatorname{Perm}(G)$. Furthermore, $N \cong G$ via the map $\lambda(g) \rho(\psi(g)) \mapsto g$.

By [7, Th. 2] we know that $N_{\psi_{1}}=N_{\psi_{2}}$ if and only if there is a fixed point free homomorphism $\zeta: G \rightarrow Z(G)$ (necessarily abelian, of course) such that $\psi_{2}(g)=$ $\psi_{1}\left(g \zeta\left(g^{-1}\right)\right) \zeta(g)$ for all $g \in G$.

As the $N$ constructed above is regular and $G$-stable, $\psi: G \rightarrow G$ gives a HopfGalois structure on $L / K$. As mentioned in [7] and explored in greater detail in [12, we have $(L[N])^{G} \cong H_{\lambda}$ as $K$-Hopf algebras, however the precise action on $L$ will be different from the canonical nonclassical action unless $\psi(G) \leq Z(G)$.

2.3. Braces and the Yang-Baxter equation. Regular, $G$-stable subgroups allow us to construct set-theoretic solutions to the Yang-Baxter equations via skew left braces.

At present time, there is not a standard notation for skew left braces. We will mostly follow the notation in [11, writing $\mathfrak{B}=(B, \cdot, \circ)$ for the skew left brace, where $(B, \cdot)$ and $(B, \circ)$ are groups and, for all $x, y, z \in B, x \circ(y \cdot z)=(x \circ y) \cdot x^{-1} \cdot(x \circ z)$. We will write $x y$ for $x \cdot y$ and $\bar{x}$ for the inverse to $x$ under $\circ$. We will refer to the operations as the dot and circle operations; some works call these the additive and multiplicative operations for historical reasons. We will denote the identity, common to both group structures, by $1_{B}$.

Skew left braces were introduced by Guarnieri and Vendramin [1], generalizing the notion of left brace formulated by Rump [17] who required that $(B, \cdot)$ be abelian. For simplicity, we will use "brace" to mean "skew left brace". We will only consider braces with $B$ finite. 
Two simple examples can be found using the group $G$ as the underlying set. We can let $\mathfrak{B}=(G, \cdot, \circ)$ where both $(G, \cdot)$ and $(G, \circ)$ are the usual group operation on $G$ (i.e., $g \cdot h=g \circ h=g h$ for all $g, h \in G$ ): we call this the trivial brace on $G$. Alternatively, we can let $\mathfrak{B}=(G, \cdot, \circ)$ with $(G, \cdot)$ the usual group operation and $g \circ h=h g$ : we call this the almost trivial brace on $G$.

Generalizing the work of [1, Byott and Vendramin [19, Prop. A.5] provide a connection between braces $(G, \cdot, \circ)$ and regular subgroups of the holomorph of $(G, \cdot)$; such subgroups gives rise to regular, $(G, \circ)$-stable subgroups, hence to HopfGalois structures on extensions will Galois group $(G, \circ)$, as follows. Suppose $(N, \cdot) \leq$ $\operatorname{Perm}(G)$ is regular and $G$-stable. Let $\varkappa: N \rightarrow G$ be the map $\varkappa(\eta)=\eta\left[1_{G}\right]$ : as $N$ is regular, $\varkappa$ is a bijection. Then $(N, \cdot, \circ)$ is a brace with $\eta \circ \pi=\varkappa^{-1}\left(\varkappa(\eta) *_{G}\right.$ $\varkappa(\pi)), \eta, \pi \in N$ where $*_{G}$ is the usual operation on $G$. The brace constructed not only incorporates $N$ (as $(N, \cdot)$ ) but also $G$ since $\varkappa:(N, \circ) \rightarrow G$ is an isomorphism. It is easy to see that $\lambda(G) \leq \operatorname{Perm}(G)$ gives the trivial brace on $G$, and $\rho(G) \leq$ $\operatorname{Perm}(G)$ gives the almost trivial brace on $G$.

If $\mathfrak{B}=(B, \cdot, \circ)$ is a brace and $(B, \cdot)$ is a nonabelian group, the notion of an opposite brace was developed independently in [18] and [15]. The opposite brace is defined as $\mathfrak{B}^{\prime}=\left(B, \cdot^{\prime}, \circ\right)$ where $x \cdot^{\prime} y=y x$. While the underlying groups of $\mathfrak{B}$ and $\mathfrak{B}^{\prime}$ are isomorphic (i.e., $(B, \cdot) \cong\left(B, \cdot^{\prime}\right)$ and, of course, $\left.(B, \circ) \cong(B, \circ)\right)$, in general $\mathfrak{B} \neq \mathfrak{B}^{\prime}$. Certainly, $\mathfrak{B}=\mathfrak{B}^{\prime}$ if and only if $(B, \cdot)$ is abelian, however there exist examples of braces isomorphic to their opposite with $(B, \cdot)$ nonabelian: see [15, Ex. 6.1]. Evidently, the trivial brace and almost trivial brace are opposites.

Braces were developed to find certain set-theoretic solutions to the Yang-Baxter equation. A set-theoretic solution to the Yang-Baxter equation is a set $B$ together with a function $R: B \times B \rightarrow B \times B$ such that

$$
(R \times \text { id })(\text { id } \times R)(R \times \text { id })=(\text { id } \times R)(R \times \text { id })(\text { id } \times R): B \times B \times B \rightarrow B \times B \times B \text {. }
$$

Writing $R(x, y)=\left(R_{x}(y), R_{y}(x)\right)$, then $R$ is non-degenerate if both $R_{x}$ and $R_{y}$ are bijections. Also, if $R(R(x, y))=(x, y)$ then $R$ is involutive.

For any brace $\mathfrak{B}=(B, \cdot, \circ)$ we let

$$
R_{\mathfrak{B}}(x, y)=\left(x^{-1}(x \circ y), \overline{x^{-1}(x \circ y)} \circ x \circ y\right)
$$

for $x, y \in B$. Then $R_{\mathfrak{B}}$ is a non-degenerate solution to the Yang-Baxter equation. Furthermore, $R_{\mathfrak{B}}$ is involutive if and only if $(B, \cdot)$ is abelian.

If $(B, \cdot)$ is nonabelian, then the opposite brace gives an additional solution

$$
R_{\mathfrak{B}^{\prime}}(x, y)=\left((x \circ y) x^{-1}, \overline{(x \circ y) x^{-1}} \circ x \circ y\right), x, y \in B ;
$$

furthermore $R_{\mathfrak{B}}$ is the inverse to $R_{\mathfrak{B}}$ (c.f, e.g., [15, Th. 4.1]).

For example, applying these constructions to the trivial brace gives

$$
R_{\mathfrak{B}}(x, y)=\left(y, y^{-1} x y\right), R_{\mathfrak{B}^{\prime}}(x, y)=\left(x y x^{-1}, x\right), x, y \in B .
$$

\section{Abelian maps AND Regular Subgroups}

We now show how to construct a regular, $G$-stable subgroup from an abelian map. Notice that we cannot simply drop the "fixed point free" condition and use Childs's construction. This is easy to see: if $\psi: G \rightarrow G$ is an abelian map and $\psi(g)=g, g \neq 1_{G}$ then $\lambda(g) \rho(\psi(g))\left[1_{G}\right]=\lambda\left(1_{G}\right) \rho\left(\psi\left(1_{G}\right)\right)\left[1_{G}\right]$ and so $\{\lambda(g) \rho(\psi(g))$ : $g \in G\}$ is not regular.

Our main result is as follows. 
Theorem 3.1. Let $\psi: G \rightarrow G$ be an abelian endomorphism. For $g \in G$, define $\eta_{g} \in \operatorname{Perm}(G)$ by $\eta_{g}[h]=g \psi\left(g^{-1}\right) h \psi(g), h \in G$, and let $N=N_{\psi}=\left\{\eta_{g}: g \in G\right\}$. Then $N$ is a regular, G-stable subgroup of $\operatorname{Perm}(G)$.

In section 4 we will show how to obtain Childs's construction from ours.

Proof. We will first show $N$ is in fact a subgroup of $\operatorname{Perm}(G)$. Note that, for $g, h, k \in G$,

$$
\begin{aligned}
\eta_{g} \eta_{k}[h] & =\eta_{g}\left[k \psi\left(k^{-1}\right) h \psi(k)\right] \\
& =g \psi\left(g^{-1}\right) k \psi\left(k^{-1}\right) h \psi(k) \psi(g) \\
& =\left(g \psi\left(g^{-1}\right) k \psi(g)\right) \psi(k g)^{-1} h \psi(k g) .
\end{aligned}
$$

Now since $\psi$ is abelian we have $\psi(k g)=\psi(g k)=\psi\left(g \psi\left(g^{-1}\right) k \psi(g)\right)$, hence

$\eta_{g} \eta_{k}[h]=\left(g \psi\left(g^{-1}\right) k \psi(g)\right) \psi\left(g \psi\left(g^{-1}\right) k \psi(g)\right)^{-1} h \psi\left(g \psi\left(g^{-1}\right) k \psi(g)\right)=\eta_{g \psi\left(g^{-1}\right) k \psi(g)}[h]$.

Thus, $\eta_{g} \eta_{k}=\eta_{g \psi\left(g^{-1}\right) k \psi(g)} \in N$. The identity in $N$ is clearly $\eta_{1_{G}}$, and $\eta_{g} \eta_{k}=$ $\eta_{g \psi\left(g^{-1}\right) k \psi(g)}=\eta_{1_{G}}$ if $k=\psi(g) g^{-1} \psi\left(g^{-1}\right)$, hence $\eta_{g}^{-1}=\eta_{\psi(g) g^{-1} \psi\left(g^{-1}\right)} \in N$ and $N \leq \operatorname{Perm}(G)$. Since $\eta_{g}\left[1_{G}\right]=g$ for all $g \in G$, given any $h, k \in G$ we have $\eta_{k} \eta_{h}^{-1}[h]=k$ and so $N$ is transitive; as the stabilizer of $1_{G}$ is trivial it follows that $N \leq \operatorname{Perm}(G)$ is regular.

Finally, we show $N$ is $G$-stable. For $g, h, k \in G$ we have

$$
\begin{aligned}
{ }^{k} \eta_{g}[h] & =\lambda(k) \eta_{g} \lambda\left(k^{-1}\right)[h] \\
& =\lambda(k) \eta_{g}\left[k^{-1} h\right] \\
& =k g \psi\left(g^{-1}\right) k^{-1} h \psi(g) \\
& =\left(k g \psi\left(g^{-1}\right) k^{-1} \psi(g)\right) \psi\left(g^{-1}\right) h \psi(g),
\end{aligned}
$$

and since $\psi\left(k g \psi\left(g^{-1}\right) k^{-1} \psi(g)\right)=\psi(g)$ we get ${ }^{k} \eta_{g}[h]=\eta_{k g \psi\left(g^{-1}\right) k^{-1} \psi(g)}[h]$ and $N$ is $G$-stable.

The opposite subgroup to $N$ is also easy to describe.

Corollary 3.2. Let $\psi$ be as above. For $g \in G$, define $\eta_{g}^{\prime} \in \operatorname{Perm}(G)$ by $\eta_{g}^{\prime}[h]=$ $h \psi\left(h^{-1}\right) g \psi(h)$, and let $N^{\prime}=N_{\psi}^{\prime}=\left\{\eta_{g}^{\prime}: g \in G\right\}$. Then $N^{\prime}$ is regular, G-stable, and $N^{\prime}=\operatorname{Cent}_{\operatorname{Perm}(G)}\left(N_{\psi}\right)$.

Proof. This can be established in a manner similar to theorem 3.1: alternatively, since $|N|=\left|N^{\prime}\right|$ it suffices to show $N$ and $N^{\prime}$ commute. Either approach is routine.

Recall that in the theory of fixed point free abelian endomorphisms it was possible to obtain the same regular, $G$-stable subgroup for two different choices of $\psi$. That remains the case here, but with a simpler criterion.

Proposition 3.3. Let $\psi_{1}, \psi_{2}: G \rightarrow G$ be abelian. Then $N_{\psi_{1}}=N_{\psi_{2}}$ if and only if there exists a homomorphism $\zeta: G \rightarrow Z(G)$ such that $\psi_{1}(g)=\zeta(g) \psi_{2}(g)$.

Remark 3.4. This is a clearer notion of equivalence than found in [7, where $\zeta$ interacts with the $\psi$ 's in a more subtle way. The condition is exactly the same: what is different is the presentation of our regular subgroup. 
Proof. For $i=1,2$ write $N_{i}=N_{\psi_{i}}=\left\{\eta_{i, g}: g \in G\right\}$. Suppose that $N_{1}=N_{2}$. Since $\eta_{1, g}\left[1_{G}\right]=\eta_{2, g}\left[1_{G}\right]=g$ we see that $\eta_{1, g}=\eta_{2, g^{\prime}}$ if and only if $g=g^{\prime}$. Thus, for all $h \in G$ we have

$$
\eta_{1, g}[h]=g \psi_{1}\left(g^{-1}\right) h \psi_{1}(g)=g \psi_{2}\left(g^{-1}\right) h \psi_{2}(g)=\eta_{2, g}[h],
$$

from which it follows that

$$
\psi_{2}(g) \psi_{1}\left(g^{-1}\right) h\left(\psi_{2}(g) \psi_{1}\left(g^{-1}\right)\right)^{-1}=h
$$

so $\psi_{2}(g) \psi_{1}\left(g^{-1}\right) \in Z(G)$. Let $\zeta(g)=\psi_{2}(g) \psi_{1}\left(g^{-1}\right)$. Then $\psi_{2}(g)=\zeta(g) \psi_{1}(g)$, and since

$$
\begin{array}{rlr}
\zeta(g h) & =\psi_{2}(g h) \psi_{1}\left((g h)^{-1}\right) & \\
& =\psi_{2}(g)\left(\psi_{2}(h) \psi_{1}\left(h^{-1}\right)\right) \psi_{1}\left(g^{-1}\right) & \\
& =\psi_{2}(g) \zeta(h) \psi_{1}\left(g^{-1}\right) & \\
& =\psi_{2}(g) \psi_{1}\left(g^{-1}\right) \zeta(h) & \\
& =\zeta(g) \zeta(h) &
\end{array}
$$

we see that $\zeta: G \rightarrow Z(G)$ is the desired homomorphism. The converse-that having such a $\zeta$ shows $N_{1}=N_{2}$-is trivial.

Remark 3.5. As is evident in the above proof, one does not need to show that $\zeta$ is a homomorphism: $N_{\psi_{1}}=N_{\psi_{2}}$ if and only if $\psi_{2}(g) \psi_{1}\left(g^{-1}\right) \in Z(G)$ for all $g \in G$.

In [16. Prop. 5.1] we show that if $\varphi \in \operatorname{Aut}(G)$ and $\psi: G \rightarrow G$ is a fixed point free abelian endomorphism, then $\varphi^{-1} \psi \varphi$ is also a fixed point free abelian endomorphism. Here, we extend this result to abelian maps, and give a condition for when conjugating by $\varphi^{-1}$ fails to give a new regular subgroup.

Proposition 3.6. If $\psi: G \rightarrow G$ is abelian, and $\varphi \in \operatorname{Aut}(G)$, then $\varphi^{-1} \psi \varphi$ is abelian. Furthermore, $N_{\psi}=N_{\varphi^{-1}} \psi \varphi$ if and only if $\psi\left(g \varphi\left(g^{-1}\right)\right) \in Z(G)$ for all $g \in G$.

Proof. Let $\psi_{\varphi}=\varphi^{-1} \psi \varphi$. That $\psi_{\varphi}$ is an abelian map is easy to show, and mimics the proof in [16, Prop. 5.1]. For the second statement, by proposition 3.3 we know $N_{\psi}=N_{\psi_{\varphi}}$ if and only if $\psi_{\varphi}(g) \psi\left(g^{-1}\right) \in Z(G)$ for all $g \in G$. We have

$$
\psi_{\varphi}(g) \psi\left(g^{-1}\right)=\left(\varphi^{-1} \psi \varphi(g)\right)\left(\psi\left(g^{-1}\right)\right)=\varphi^{-1} \psi\left(\varphi(g) g^{-1}\right),
$$

which is in $Z(G)$ if and only if $\psi\left(\varphi(g) g^{-1}\right) \in Z(G)$, which is true if and only if its inverse, $\psi\left(g \varphi\left(g^{-1}\right)\right)$, is in $Z(G)$.

Let us consider some examples. These are generalizations of examples presented in 14$]$.

Example 3.7. Let $n \geq 5$, and suppose $\psi: S_{n} \rightarrow S_{n}$ is abelian. Since ker $\psi \triangleleft S_{n}$ we must have $\operatorname{ker} \psi=\left\{1_{S_{n}}\right\}, A_{n}$, or $S_{n}$. As $\psi$ is abelian we know that ker $\psi \neq\left\{1_{S_{n}}\right\}$, and ker $\psi=S_{n}$ if and only if $\psi$ is the trivial map. We shall assume ker $\psi=A_{n}$.

Since $S_{n}$ is generated by transpositions it suffices to describe $\psi(\tau)$ for all transpositions $\tau$. Furthermore, since $\psi$ is abelian and all transpositions are conjugate, $\psi\left(\tau_{1}\right)=\psi\left(\tau_{2}\right)$ for all transpositions $\tau_{1}, \tau_{2} \in S_{n}$. Since $\tau^{2} \in A_{n}$ we know $\psi(\tau)$ has order 2. So let $\xi \in S_{n}$ have order 2 , and define

$$
\psi(\sigma):=\psi_{\xi}(\sigma)=\left\{\begin{array}{ll}
1_{G} & \sigma \in A_{n} \\
\xi & \sigma \notin A_{n}
\end{array} .\right.
$$


This is clearly an endomorphism, and since $\psi\left(S_{n}\right)=\langle\xi\rangle \cong C_{2}$ it is abelian. We can see that $\psi$ is fixed point free if and only if $\xi \in A_{n}$. The corresponding regular subgroup is $N=\left\{\eta_{\sigma}: \sigma \in S_{n}\right\}$ with

$$
\eta_{\sigma}[\pi]=\left\{\begin{array}{ll}
\sigma \pi & \sigma \in A_{n} \\
\sigma \xi \pi \xi & \sigma \notin A_{n}
\end{array} .\right.
$$

Since $Z\left(S_{n}\right)$ is trivial, each choice of $\xi$ produces a different regular, $G$-stable subgroup. Note that if we extend the choices of $\xi$ to include $\xi=1_{G}$ we also have the trivial map in this classification.

We can also compute the elements of the opposite subgroup: $N^{\prime}=\left\{\eta_{\sigma}^{\prime}: \sigma \in G\right\}$ with

$$
\eta_{\sigma}^{\prime}[\pi]=\left\{\begin{array}{ll}
\pi \sigma & \pi \in A_{n} \\
\pi \xi \sigma \xi & \pi \notin A_{n}
\end{array} .\right.
$$

We will see later that the subgroups above capture all of the regular, $S_{n}$-stable subgroups of $S_{n}$ in the case $n=5$.

Example 3.8. Let $p>q$ be primes, $p \equiv 1(\bmod q)$, and let $M_{p, q}$ denote the nonabelian group of order $p q$, namely

$$
M_{p, q}=\left\langle s, t: s^{p}=t^{q}=1_{G}, t s t^{-1}=s^{d}\right\rangle
$$

where $d$ is an integer whose (multiplicative) order is $q \bmod p$. If $\psi: M_{p, q} \rightarrow M_{p, q}$ is a nontrivial abelian endomorphism then $\operatorname{ker} \psi=\langle s\rangle$ since the Sylow $p$-subgroup is normal in $M_{p, q}$ and the Sylow $q$-subgroup is not. Thus $\psi(s)=1_{M_{p, q}}$. Write

$$
\psi(t)=s^{i} t^{j}, 0 \leq i \leq p-1,1 \leq j \leq q-1 .
$$

Then $\psi_{i, j}:=\psi$ is an endomorphism with cyclic image, hence abelian. As observed in [14, 6.6], $\psi$ is fixed point free if and only if $j \neq 1$. We will see later that all regular, $M_{p, q}$-stable subgroups of $M_{p, q}$ come from abelian maps, either directly or through the opposite construction.

\section{Fixed POINT FREE ABELIAN ENDOMORPHisms AND BEyOND}

Here, we will show how our work includes all of the constructions in [7, where the abelian maps are all fixed point free. We will also provide a class of examples which suggests that our construction encompasses significantly more Hopf-Galois structures. Recall that an abelian map $\Psi: G \rightarrow G$ is fixed point free if $\Psi(g)=g$ implies $g=1_{G}$. (Note the slight change in notation, reserving $\psi$ for our abelian maps.)

Recall that a fixed point free abelian map $\Psi: G \rightarrow G$ gives rise to a regular, $G$-stable subgroup $N=\{\lambda(g) \rho(\Psi(g)): g \in G\}$ which is isomorphic to $G$. As a consequence,

$$
N\left[1_{G}\right]=\left\{g \Psi\left(g^{-1}\right): g \in G\right\}=G,
$$

so every $k \in G$ can be represented uniquely as $k=g \psi\left(g^{-1}\right)$ for some $g \in G$. Define $\psi: G \rightarrow G$ by $\psi\left(g \Psi\left(g^{-1}\right)\right)=\Psi\left(g^{-1}\right)$. We first claim that $\psi$ is a fixed point free abelian endomorphism. (Indeed, it is the quasi-inverse of $\Psi$ as described 
in [7] and [3].) Note that

$$
\begin{aligned}
& \left(g \Psi\left(g^{-1}\right) h \Psi(g)\right) \Psi\left(g \Psi\left(g^{-1}\right) h \Psi(g)\right)^{-1} \\
& \quad=\left(g \Psi\left(g^{-1}\right) h \Psi(g)\right) \Psi\left(h^{-1} g^{-1}\right)=g \Psi\left(g^{-1}\right) h \Psi\left(h^{-1}\right),
\end{aligned}
$$

so

$$
\begin{aligned}
\psi\left(g \Psi\left(g^{-1}\right) h \Psi\left(h^{-1}\right)\right) & =\psi\left(\left(g \Psi\left(g^{-1}\right) h \Psi(g)\right) \Psi\left(g \Psi\left(g^{-1}\right) h \Psi(g)\right)^{-1}\right) \\
& =\Psi\left(g \Psi\left(g^{-1}\right) h \Psi(g)\right)^{-1} \\
& =\Psi\left(g^{-1} h^{-1}\right) \\
& =\Psi\left(g^{-1}\right) \Psi\left(h^{-1}\right) \\
& =\psi\left(g \Psi\left(g^{-1}\right)\right) \psi\left(h \Psi\left(h^{-1}\right)\right)
\end{aligned}
$$

and $\psi: G \rightarrow G$ is a homomorphism.

Next, if $\psi\left(g \Psi\left(g^{-1}\right)\right)=g \Psi\left(g^{-1}\right)$ then $g=1_{G}$ by the definition of $\psi$, hence $\psi$ is fixed point free. Additionally, $\psi(G)=\Psi(G)$ so $\psi$ is abelian.

Next we claim that, for all $g \in G, \eta_{g \Psi\left(g^{-1}\right)}=\lambda(g) \rho(\Psi(g))$. Indeed, we have

$$
\begin{aligned}
\eta_{g \Psi\left(g^{-1}\right)}[h] & =\left(g \Psi\left(g^{-1}\right)\right) \psi\left(\left(g \Psi\left(g^{-1}\right)\right)^{-1}\right) h \psi\left(g \Psi\left(g^{-1}\right)\right) \\
& =g \Psi\left(g^{-1}\right) \Psi(g) h \Psi\left(g^{-1}\right) \\
& =\lambda(g) \rho(\Psi(g))[h] .
\end{aligned}
$$

Thus, the construction presented in this work includes all of the structures found in 7 .

Conversely, if $\psi: G \rightarrow G$ is fixed point free abelian, giving the regular, $G$-stable subgroup $N=\left\{\eta_{g}\right\}$ as above, defining $\Psi: G \rightarrow G$ by $\Psi\left(g \psi\left(g^{-1}\right)\right)=\psi\left(g^{-1}\right)$ gives a fixed point free abelian map, and

$$
\lambda\left(g \psi\left(g^{-1}\right)\right) \rho\left(\Psi\left(g \psi\left(g^{-1}\right)\right)\right)[h]=g \psi\left(g^{-1}\right) h \Psi\left(\psi(g) g^{-1}\right)=g \psi\left(g^{-1}\right) h \psi(g)=\eta_{g}[h] .
$$

Thus, there is a one-to-one correspondence between the fixed point free constructions presented here and the constructions in [7.

The following "normal complement" example gives a completely different family of Hopf-Galois structures obtained through abelian maps.

Example 4.1. Let $G^{\prime}, G^{\prime \prime} \leq G$ with $G^{\prime} \triangleleft G, G^{\prime \prime}$ abelian, $G^{\prime} \cap G^{\prime \prime}=\left\{1_{G}\right\}$, and $\left|G^{\prime}\right|\left|G^{\prime \prime}\right|=|G|$. Define $\psi: G \rightarrow G$ by $\psi(h k)=k, h \in G^{\prime}, k \in G^{\prime \prime}$. As $G^{\prime}$ is normal in $G, \psi$ is a homomorphism, evidently abelian. The corresponding regular, $G$-stable subgroup of $\operatorname{Perm}(G)$ can be made quite explicit: $N=\left\{\eta_{h k}: h \in G^{\prime}, k \in G^{\prime \prime}\right\}$ with $\eta_{h k}=\lambda(h) \rho(k)$. Thus, $N \cong G^{\prime} \times G^{\prime \prime}$.

One obtains from this a proof of [8, Cor. 6] (see also [5, Cor. 7.2]) in the case where the (potentially) non-normal group (here, $G^{\prime \prime}$ ) is abelian.

Note that if $G^{\prime}$ is nonabelian then we have the opposite subgroup $N^{\prime}=\left\{\eta_{h k}^{\prime}\right.$ : $\left.h \in G^{\prime}, k \in G^{\prime \prime}\right\}$ with $\eta_{h k}^{\prime}[x y]=x y \psi\left((x y)^{-1}\right) h k \psi(x y)=x h k y^{-1}, x \in G^{\prime}, y \in G^{\prime \prime}$ as can be readily computed.

Returning to example 3.7, where an abelian map $\psi: S_{n} \rightarrow S_{n}, n \geq 5$ depends on the choice of a $\xi \in S_{n}$ with $\xi^{2}=1_{S_{n}}$, we observed that $\psi$ was fixed point free if and only if $\xi \in A_{n}$. Now, observe that if $\xi \notin A_{n}$ then $S_{n}=A_{n}\langle\xi\rangle$ and the above applies, producing a Hopf-Galois structure of type $A_{n} \times C_{2}$. In [4, Th. 5, Th. 9] 
there is a complete description of regular, $G$-stable subgroups of $\operatorname{Perm}\left(S_{n}\right), n \geq 5$ of type $S_{n}$ as well as of type $A_{n} \times C_{2}$. The classification given here, together with the opposite groups, account for all such subgroups. Furthermore, 9, Prop. 5] states that the only regular, $S_{5}$-stable subgroups of $\operatorname{Perm}\left(S_{5}\right)$ are of type $S_{5}$ or $A_{5} \times C_{2}$. Thus abelian maps give us all desired subgroups when $n=5$.

Similarly, in example 3.8 an abelian map $\psi: M_{p, q} \rightarrow M_{p, q}$ depends on integers $0 \leq i \leq p-1,1 \leq j \leq q-1$; furthermore $\psi$ is fixed point free if and only if $j \neq 1$. The case $j=1$ can be interpreted using example 4.1 by writing $M_{p, q}=\langle s\rangle\left\langle s^{i} t\right\rangle$, giving a Hopf-Galois structure of type $C_{p} \times C_{q}$. The Hopf-Galois structures on a metacyclic extension are fully described in [2]. They are all of type $M_{p, q}$ or $C_{p} \times C_{q}$. Using the characterization found in [16, §8] it is clear that we have found all such structures here (once opposites are considered in the type $M_{p, q}$ case).

\section{Abelian maps And BRaces}

In [14, Prop. 4.4] the brace corresponding to a fixed point free abelian endomorphism is found. Here we will duplicate this result while allowing our abelian map to have fixed points.

In fact, our description of the regular, $G$-stable subgroup $N:=N_{\psi}$ (for $\psi: G \rightarrow$ $G$ abelian) allows for a simpler proof than the one given in [14. The primary reason for this is that the map $\varkappa: N \rightarrow G$ is particularly nice using our construction: $\varkappa\left(\eta_{g}\right)=\eta_{g}\left[1_{G}\right]=g$. Recall that $\eta_{g} \eta_{h}=\eta_{g \psi\left(g^{-1}\right) h \psi(g)}$ and $\eta_{g}^{-1}=\eta_{\psi(g) g^{-1} \psi\left(g^{-1}\right)}$. If we define $\eta_{g} \circ \eta_{h}=\varkappa^{-1}(\varkappa(g) \varkappa(h))=\varkappa^{-1}(g h)=\eta_{g h}$ then $(N, \cdot, \circ)$ is a brace.

It would seem desirable to think of our underlying set as $G$ instead of $N$. If we identify, through $\varkappa$, the elements of $N$ with the elements of $G$, then the dot operation becomes $g \cdot h=\varkappa\left(\eta_{g} \eta_{h}\right)=\varkappa\left(\eta_{g \psi\left(g^{-1}\right) h \psi(g)}\right)=g \psi\left(g^{-1}\right) h \psi(g)$ and the circle operation becomes $g \circ h=\varkappa\left(\eta_{g} \circ \eta_{h}\right)=\varkappa\left(\eta_{g h}\right)=g h$. This creates an inconvenient issue with notation, as we can no longer suppress the dot expressions without creating confusion. However, this can be remedied: we claim that $(G, \circ, \cdot)$ is also a brace, which we can prove by showing the brace relation holds on $(G, \circ, \cdot)$. Swapping our operations this way, for $g, h, k \in G$ we need to show $g \cdot(h \circ k)=$ $(g \cdot h) \circ \bar{g} \circ(g \cdot k)$. We have

$$
\begin{aligned}
(g \cdot h) \circ \bar{g} \circ(g \cdot k) & =\left(g \psi\left(g^{-1}\right) h \psi(g)\right) \circ \bar{g} \circ\left(g \psi\left(g^{-1}\right) k \psi(g)\right) \\
& =\left(g \psi\left(g^{-1}\right) h \psi(g)\right) g^{-1}\left(g \psi\left(g^{-1}\right) k \psi(g)\right) \\
& =g \psi\left(g^{-1}\right) h k \psi(g)=g \circ(h k) .
\end{aligned}
$$

The brace constructed above is what Childs, in [5] calls a bi-skew brace. In short, a bi-skew brace is any (skew left) brace where interchanging the operations results in another (skew left) brace. Noticing that our brace is bi-skew allows us to choose which is the dot operation. For reasons mentioned above, it seems reasonable to exchange the operations, giving the following.

Proposition 5.1. Let $\psi$ be an abelian map on $(G, \cdot)$. Then $\mathfrak{B}_{\psi}=(G, \cdot, \circ)$ is a bi-skew brace, with $g \cdot h=g h$ and $g \circ h=g \psi\left(g^{-1}\right) h \psi(g)$. Furthermore, $(G, \circ) \cong N_{\psi}$.

Of course, this can also be verified by simply checking $(G, \circ)$ is a group and the brace relation holds. The brace constructed is identical to the brace in [14] in the fixed point free case.

The observation that $\mathfrak{B}_{\psi}$ is bi-skew allows us to construct another Hopf-Galois structure on a Galois extension with, potentially, a different Galois group. In order 
to minimize confusion below we will adopt very explicit notation for all of our binary operations.

Theorem 5.2. Let $\psi:(G, \cdot) \rightarrow(G, \cdot)$ be an abelian endomorphism, and define $(G, \circ)$ as above. Suppose $\left(N, *_{N}\right)$ is an abstract group which is isomorphic to $(G, \circ)$. Then $\psi$ gives rise to a regular, $N$-stable subgroup $P \leq \operatorname{Perm}(N)$ with $P \cong G$.

Proof. Let $\alpha:(G, \circ) \rightarrow\left(N, *_{N}\right)$ be an isomorphism. For $g \in G$, define $\pi_{g}: N \rightarrow N$ by $\pi_{g}[n]=\alpha\left(g \cdot \alpha^{-1}(n)\right)$, and let $P=\left\{\pi_{g}: g \in G\right\}$. Then

$$
\begin{aligned}
\pi_{g} \pi_{h}[n] & =\pi_{g}\left[\alpha\left(h \cdot \alpha^{-1}(n)\right)\right] \\
& =\alpha\left(g \cdot \alpha^{-1}\left(\alpha(h) *_{N} n\right)\right) \\
& =\alpha\left(g \cdot h \cdot \alpha^{-1}(n)\right)=\pi_{g \cdot h}[n],
\end{aligned}
$$

so $P \leq \operatorname{Perm}(N)$ and $P \cong G$. One can easily show $P$ is regular.

Furthermore, we have

$$
\begin{aligned}
{ }^{m} \pi_{g}[n] & =\lambda(m) \pi_{g} \lambda\left(m^{-1}\right)[n] \\
& =m *_{N} \alpha\left(g \cdot \alpha^{-1}\left(m^{-1} *_{N} n\right)\right) \\
& =m *_{N} \alpha\left(\alpha^{-1}(\alpha(g)) \cdot \alpha^{-1}\left(m^{-1} *_{N} n\right)\right) \\
& =m \alpha\left(\alpha^{-1}\left(\alpha(g) *_{N} m^{-1} *_{N} n\right)\right) \\
& =m *_{N} \alpha(g) *_{N} m^{-1} *_{N} n,
\end{aligned}
$$

whereas

$$
\begin{aligned}
\pi_{\alpha^{-1}(m) \cdot g \cdot \alpha^{-1}\left(m^{-1}\right)}[n] & =\alpha\left(\alpha^{-1}(m) \cdot g \cdot \alpha^{-1}\left(m^{-1}\right) \cdot \alpha^{-1}(n)\right) \\
& =\alpha\left(\alpha^{-1}(m) \cdot \alpha^{-1}(\alpha(g)) \cdot \alpha^{-1}\left(m^{-1}\right) \cdot \alpha^{-1}(n)\right) \\
& =m *_{N} \alpha(g) *_{N} m^{-1} *_{N} n,
\end{aligned}
$$

hence ${ }^{m} \pi_{g}=\pi_{\alpha^{-1}(m) \cdot g \cdot \alpha^{-1}\left(m^{-1}\right)}$ and $P$ is $N$-stable.

Example 5.3. Let $G=S_{n}, \xi \in S_{n}$ an odd permutation of order 2, and suppose $n \geq 5$. Let $\psi=\psi_{\xi}$ as in example 3.7. Then we have seen that $N_{\psi} \cong A_{n} \times C_{2}$, hence there is be a Hopf-Galois structure on an $A_{n} \times C_{2}$ extension of type $S_{n}$. An isomorphism $\alpha$ : $(G, \circ) \rightarrow A_{n} \times C_{2}$ corresponding to our choice of $\xi$ is given by $\alpha(\sigma)=\left(\sigma, 1_{C_{2}}\right), \alpha(\tau)=(\tau \xi, \xi), \sigma \in A_{n}, \tau \notin A_{n}, C_{2}=\langle\xi\rangle$. Then

$$
\pi_{\tau}\left[\left(\sigma, 1_{C_{2}}\right)\right]=\left\{\begin{array}{ll}
\left(\tau \sigma, 1_{C_{2}}\right) & \tau \in A_{n} \\
(\tau \sigma \xi, \xi) & \tau \notin A_{n}
\end{array}, \pi_{\tau}[(\sigma, \xi)]=\left\{\begin{array}{ll}
\left(\tau \sigma, 1_{C_{2}}\right) & \tau \in A_{n} \\
(\tau \sigma, \xi) & \tau \notin A_{n}
\end{array} .\right.\right.
$$

Of course, the construction above depends on a choice of isomorphism $\alpha$ : $(G, \circ) \rightarrow N$. If $\beta:(G, \circ) \rightarrow N$ is another isomorphism, then $\alpha \beta^{-1} \in \operatorname{Aut}(N)$ and the resulting regular subgroups differ by conjugation by $\alpha \beta^{-1}$.

We have described how one can use a brace $(B, \cdot, \circ)$ to construct two set-theoretic solutions to the Yang-Baxter equation. When a brace is in fact bi-skew, we get (up 
to) four solutions, namely

$$
\begin{aligned}
& R_{\mathfrak{B}}(x, y)=\left(x^{-1}(x \circ y), \overline{x^{-1}(x \circ y)} \circ x \circ y\right) \\
& R_{\mathfrak{B}^{\prime}}(x, y)=\left((x \circ y) x^{-1}, \overline{(x \circ y) x^{-1}} \circ x \circ y\right) \\
& S_{\mathfrak{B}}(x, y)=\left(\bar{x} \circ(x y),(\bar{x} \circ(x y))^{-1} x y\right) \\
& S_{\mathfrak{B}^{\prime}}(x, y)=\left((x y) \circ \bar{x},((x y) \circ \bar{x})^{-1} x y\right) .
\end{aligned}
$$

Applying these formulas to our bi-skew brace gives the following.

Corollary 5.4. Let $\psi: G \rightarrow G$ be an abelian endomorphism. Then each of the following is a set-theoretic solution to the Yang-Baxter equation:

$$
\begin{aligned}
& R_{1, \psi}(g, h)=\left(\psi\left(g^{-1}\right) h \psi(g), \psi\left(h g^{-1}\right) h^{-1} \psi(g) g \psi\left(g^{-1}\right) h \psi\left(g h^{-1}\right)\right) \\
& R_{2, \psi}(g, h)=\left(g \psi\left(g^{-1}\right) h \psi(g) g^{-1}, \psi(h) g \psi\left(h^{-1}\right)\right) \\
& R_{3, \psi}(g, h)=\left(\psi(g) h \psi\left(g^{-1}\right), \psi(g) h^{-1} \psi\left(g^{-1}\right) g h\right) \\
& R_{4, \psi}(g, h)=\left(g h \psi\left(h^{-1}\right) g^{-1} \psi(h), \psi\left(h^{-1}\right) g \psi(h)\right), g, h \in G .
\end{aligned}
$$

Furthermore:

(1) $R_{1, \psi}=R_{2, \psi}$ if and only if $G$ is an abelian group (in which case $R_{1, \psi}(g, h)=$ $\left.R_{2, \psi}(g, h)=(h, g)\right)$.

(2) $R_{3 . \psi}=R_{4, \psi}$ if and only if $g \psi\left(g^{-1}\right) h \psi(g)=h \psi\left(h^{-1}\right) g \psi(h)$ for all $g, h \in G$.

(3) $R_{1, \psi} R_{2, \psi}=R_{3, \psi} R_{4, \psi}=$ id.

Proof. The solutions are straightforward computations, and (1)-(3) follow from properties of opposite braces.

\section{Five subgroups of $G$ And $N$, And five sub-Hopf-Algebras of $K[N]^{G}$}

Recall that if $\psi: G \rightarrow G$ is a fixed point free abelian map, then we obtain a Hopf Galois structure of type $G$ whose Hopf algebra $L[N]^{G}$ is isomorphic to $H_{\lambda}$ as $K$-Hopf algebras. Once we allow $\psi$ to have fixed points our structure may no longer be of type $G$, nor need the Hopf algebra be isomorphic to $H_{\lambda}$. We ask: can we determine the type of the Hopf-Galois structure arising from an abelian map $\psi$ ? In general, there appears to be no easy way to determine the isomorphism type of $N_{\psi}$, however we are able to obtain some results about this group's structure.

We will investigate questions on Hopf-Galois structure through the use of five subgroups of $G$ that depend on $\psi$. Each of these subgroups give rise to a (nonregular) $G$-stable subgroup of $\operatorname{Perm}(G)$, which in turn will give a sub-Hopf algebra of $H=L[N]^{G}$.

In [16, the concepts of $\lambda$-points and $\rho$-points were introduced to investigate questions involving brace equivalence. Given a regular, $G$-stable subgroup $N \leq$ $\operatorname{Perm}(G)$ the sets of $\lambda$-points and $\rho$-points, denoted $\Lambda_{N}$ and $P_{N}$ respectively, are defined as

$$
\Lambda_{N}=N \cap \lambda(G), P_{N}=N \cap \rho(G) .
$$

Both $\Lambda_{N}$ and $P_{N}$ are subgroups of $N$. Note that if $N_{1}$ and $N_{2}$ are brace equivalent then $\Lambda_{N_{1}} \cong \Lambda_{N_{2}}$ and $P_{N_{1}} \cong P_{N_{2}}$ [16, Prop. 6.3].

First, we let $G_{0}=\operatorname{ker} \psi, N_{0}=\left\{\eta_{g_{0}}: g_{0} \in G_{0}\right\}$. Since $\eta_{g_{0}}[h]=g_{0} \psi\left(g_{0}^{-1}\right) h \psi\left(g_{0}\right)=$ $g_{0} h, g_{0} \in G_{0}, h \in G$ we have $N_{0}=\lambda\left(G_{0}\right) \leq N$. In particular, note that $N_{0} \cong G_{0}$, 
providing us some information as to the structure of $N$. Clearly, $G_{0} \triangleleft G$, and

$$
\begin{aligned}
\eta_{g} \eta_{g_{0}} \eta_{g}^{-1} & =\eta_{g} \eta_{g_{0} \psi\left(g_{0}^{-1}\right)\left(\psi(g) g^{-1} \psi\left(g^{-1}\right)\right) \psi\left(g_{0}\right)} \\
& =\eta_{g} \eta_{g_{0} \psi(g) g^{-1}} \psi\left(g^{-1}\right) \\
& =\eta_{g \psi\left(g^{-1}\right)\left(g_{0} \psi(g) g^{-1} \psi\left(g^{-1}\right)\right) \psi(g)} \\
& =\eta_{\left(g \psi\left(g^{-1}\right)\right) g_{0}\left(g \psi\left(g^{-1}\right)\right)^{-1}} \in N_{0}
\end{aligned}
$$

by the normality of $G_{0}$.

Since $N_{0}=\lambda\left(G_{0}\right)$ is $G$-stable, by [10, Th. 5.2] (see also [13, Prop. 2.2] for a more explicit formulation), $H_{0}:=\left(L\left[N_{0}\right]\right)^{G}=L\left[\lambda\left(G_{0}\right)\right]^{G}$ is a sub-Hopf algebra of $H$ which is also contained in $H_{\lambda}$.

Generalizing slightly, let $\widehat{G}_{0}=\psi^{-1}(Z(G))$. This is evidently a subgroup of $G$ containing $G_{0}$, and let $\widehat{N}_{0}:=\left\{\eta_{\hat{g}_{0}}: \hat{g}_{0} \in \widehat{G}_{0}\right\}$.

Lemma 6.1. With notation as above, $\widehat{N}_{0}=\Lambda_{N}$.

Proof. For $\hat{g}_{0} \in \widehat{G}_{0}, h \in G$ we have $\eta_{\hat{g}_{0}}[h]=\hat{g}_{0} \psi\left(\hat{g}_{0}^{-1}\right) h \psi\left(\hat{g}_{0}\right)=\hat{g}_{0} h$ so clearly $\widehat{N}_{0} \subset \Lambda_{N}$. Conversely, if $\eta_{g} \in \Lambda_{N}$ then $\eta_{g}=\lambda(k)$ for some $k \in G$ : evaluating this expression at $1_{G}$ shows $g=k$. Thus, $\eta_{g}[h]=g \psi\left(g^{-1}\right) h \psi(g)=g h$, so $\psi\left(g^{-1}\right) h \psi(g)=h$ for all $h \in G$. This can only occur if $\psi(g) \in Z(G)$.

As both $N$ and $\lambda(G)$ are $G$-stable, so is $\widehat{N}_{0}$. Thus, $\widehat{H}_{0}:=\left(L\left[\widehat{N}_{0}\right]\right)^{G}$ is a sub-Hopf algebra of $H$. It is precisely the sub-Hopf algebra of $H_{\lambda}$ obtained by restricting to $\widehat{G}_{0}$, i.e., $\widehat{H}_{0}=L\left[\lambda\left(\widehat{G}_{0}\right)\right]^{G}$.

Next, let $G_{1}=\left\{g_{1} \in G: \psi\left(g_{1}\right)=g_{1}\right\}$ be the subgroup of fixed points. Clearly $G_{1}$ is abelian, although typically $G_{1}$ is not normal in $G$. If we let $N_{1}=\left\{\eta_{g_{1}}: g_{1} \in G_{1}\right\}$ then $\eta_{g_{1}}[h]=g_{1} g_{1}^{-1} h g_{1}=h g_{1}$ for all $h \in G$, hence $\eta_{g_{1}}=\rho\left(g_{1}^{-1}\right)$. Thus $N_{1}=\rho\left(G_{1}\right)$ is a subgroup of $N$ isomorphic to $G_{1}$.

We also have ${ }^{k} \eta_{g_{1}}=\eta_{k g_{1} \psi\left(g_{1}^{-1}\right) k^{-1} \psi\left(g_{1}\right)}=\eta_{\psi\left(g_{1}\right)}$, so $G$ acts trivially on $N_{1}$. Thus, $H_{1}=\left(L\left[N_{1}\right]\right)^{G}=K\left[G_{1}\right]$ is a sub-Hopf algebra of $H$.

Generalizing again, let $\phi: G \rightarrow G$ be given by $\phi(g)=g \psi\left(g^{-1}\right)$ for all $g \in G$. Define $\widehat{G}_{1}=\{\hat{g} \in G: \phi(\hat{g}) \in Z(G)\}$. As $\phi$ is trivial on fixed points we clearly have $G_{1} \leq \widehat{G}_{1}$. Since $\phi(\hat{g} \hat{h})=\hat{g} \hat{h} \psi\left(\hat{h}^{-1} \hat{g}^{-1}\right)=\hat{g} \phi(\hat{h}) \psi\left(\hat{g}^{-1}\right)=\phi(\hat{g}) \phi(\hat{h})$ for $\hat{h} \in \widehat{G}_{1}$ we see this is in fact a subgroup of $G$. If we define $\widehat{N}_{1}=\left\{\eta_{\hat{g}_{1}}: \hat{g}_{1} \in \widehat{G}_{1}\right\}$ we get

Lemma 6.2. With notation as above, $\widehat{N}_{1}=P_{N}$.

Proof. For $\hat{g}_{1} \in \widehat{G}_{1}$ we have $\eta_{\hat{g}_{1}}[h]=\hat{g}_{1} \psi\left(\hat{g}_{1}^{-1}\right) h \psi\left(\hat{g}_{1}\right)=\phi\left(\hat{g}_{1}\right) h \psi\left(\hat{g}_{1}\right)=h \phi\left(\hat{g}_{1}\right) \psi\left(\hat{g}_{1}\right)$ $=h \hat{g}_{1}$ hence $\eta_{\hat{g}_{1}}=\rho\left(\hat{g}_{1}^{-1}\right) \in P_{N}$. Conversely, suppose $\eta_{g}=\rho(k)$. By evaluating at $1_{G}$ we see $k=g^{-1}$, hence for all $h \in G$ we have

$$
\begin{aligned}
\eta_{g}[h]=g \psi\left(g^{-1}\right) h \psi(g) & =h g \\
g \psi\left(g^{-1}\right) h & =h g \psi\left(g^{-1}\right) \\
\phi(g) h & =h \phi(g),
\end{aligned}
$$

thus $\phi(g) \in Z(G)$, i.e., $g \in \widehat{G}_{1}$. Therefore, $\eta_{g} \in \widehat{N}_{1}$.

Since ${ }^{k} \eta_{\hat{g}_{1}}=\eta_{k \phi\left(\hat{g}_{1}\right) k^{-1} \psi\left(\hat{g}_{1}\right)}=\eta_{\hat{g}_{1}}$ we see that $G$ acts trivially on $\widehat{N}_{1}$. Then $\widehat{H}_{1}:=(L[\widehat{N}])^{G}=K\left[\widehat{G}_{1}\right]$ is a sub-Hopf algebra of $H$. In fact, $\widehat{H}_{1}$ is the largest group ring contained in $H$. 
Finally, note that $G_{0} \cap G_{1}$ is trivial, hence $N_{0} \cap N_{1}=\left\{1_{N}\right\}$. Since $G_{0} \triangleleft G$ and $N_{0} \triangleleft N$ we get

$$
\begin{aligned}
& G_{01}:=G_{0} G_{1}=\left\{g_{0} g_{1}: g_{0} \in G_{0}, g_{1} \in G_{1}\right\} \leq G, \\
& N_{01}:=N_{0} N_{1}=\left\{\eta_{g_{0} g_{1}}: g_{0} \in G_{0}, g_{1} \in G_{1}\right\} \leq N .
\end{aligned}
$$

We have $\eta_{g_{0} g_{1}}[h]=g_{0} g_{1} \psi\left(g_{1}^{-1} g_{0}^{-1}\right) h \psi\left(g_{0} g_{1}\right)=g_{0} h g_{1}$ hence $\eta_{g_{0} g_{1}}=\lambda\left(g_{0}\right) \rho\left(g_{1}^{-1}\right)$. The group $N_{01}$ is evidently $G$-stable, giving rise to another sub-Hopf algebra $H_{01}:=$ $\left(L\left[N_{01}\right]\right)^{G} \cong\left(L\left[N_{0}\right]\right)^{G} \otimes K\left[G_{1}\right]$.

The construction of the subgroups above allow us to obtain the following. Note the relationship between the following result and example 4.1.

Proposition 6.3. Let $\psi: G \rightarrow G$ be abelian, and let $G_{0}, G_{1}, N_{0}, N_{1}$ be as above. Then $N_{\psi}$ has a subgroup isomorphic to $G_{0} \times G_{1}$. In particular, if $\left|G_{0}\right|\left|G_{1}\right|=|G|$ then $N_{\psi} \cong G_{0} \times G_{1}$.

Proof. The work above creates the subgroup $N_{0,1}=N_{0} N_{1}$. That $N_{0,1} \cong N_{0} \times N_{1}$ follows from $\eta_{g_{0} g_{1}} \eta_{h_{0} h_{1}}=\lambda\left(g_{0}\right) \rho\left(g_{1}^{-1}\right) \lambda\left(h_{0}\right) \rho\left(h_{1}^{-1}\right)=\lambda\left(g_{0} h_{0}\right) \rho\left(g_{1} h_{1}\right)=\eta_{g_{0} h_{0} g_{1} h_{1}}$.

We conclude with an investigation of dihedral groups. Let $G=D_{n}=\langle r, s$ : $r^{n}=s^{2}=$ rsrs $\left.=1_{G}\right\rangle$. We will find all abelian maps on $G$ and determine the type of each Hopf-Galois structure. Suppose $\psi: G \rightarrow G$ is an abelian map. Since $\psi(r) \psi(s)=\psi(r s)=\psi(s r)=\psi\left(r^{-1} s\right)=\psi\left(r^{-1}\right) \psi(s)$ we know that $\psi(r)$ must be an element whose order divides both 2 and $n$. We will examine two cases, based on the parity of $n$.

Suppose first that $n$ is odd. Then $\psi(r)=1_{G}$. Letting $\psi(s)=r^{i} s$ for some $0 \leq i \leq n-1$ gives an abelian map, and it is clear that every nontrivial abelian map is of this form. Since $Z\left(D_{n}\right)=\left\{1_{G}\right\}$ each choice of $i$ gives a different regular, $G$-stable subgroup. For each, $G_{0}=\langle r\rangle$ and $G_{1}=\left\langle r^{i} s\right\rangle$, so the resulting Hopf-Galois structure is of type $C_{n} \times C_{2}$. Thus we have $n+1$ Hopf-Galois structures, one of type $D_{n}$ and $n$ of type $C_{n} \times C_{2}$.

Now suppose $n$ is even. Then $\psi(r)=1_{G}, r^{n / 2}$, or $r^{i} s$ for some $0 \leq i \leq n-1$; and $\psi(s)=1_{G}, r^{n / 2}$, or $r^{j} s$ for some $0 \leq j \leq n-1$. However, since $r^{n / 2} \in Z\left(D_{n}\right)$ we need only study the following cases:

Case $1\left(\psi(r)=\psi(s)=1_{G}\right)$. Then $\psi=\mathrm{id}$, and the Hopf-Galois structure is of type $D_{n}$.

Case $2\left(\psi(r)=1_{G}, \psi(s)=r^{j} s, 0 \leq j \leq(n / 2)-1\right)$. As in the case $n$ is odd we get $G_{0}=\langle r\rangle$ and $G_{1}=\left\langle r^{j} s\right\rangle$, hence we get $n / 2$ Hopf-Galois structures of type $C_{n} \times C_{2}$.

Case $3\left(\psi(r)=r^{i} s, \psi(s)=1_{G}, 0 \leq i \leq(n / 2)-1\right)$. Since $\psi\left(r^{i} s\right)=\left(r^{i} s\right)^{i}$ we see that $r^{i} s$ is a fixed point if an only if $i$ is odd. If $i$ is even then $\psi$ is fixed point free, hence the corresponding Hopf-Galois structure is of type $D_{n}$. On the other hand, if $i$ is odd, then $G_{0}=\left\langle r^{2}, s\right\rangle \cong D_{n / 2}$ (note $D_{2} \cong C_{2} \times C_{2}$ ) and $G_{1}=\left\langle r^{i} s\right\rangle$, hence the Hopf-Galois structure is of type $D_{n / 2} \times C_{2}$. Overall, this case gives $n / 4$ HopfGalois structures of type $D_{n}$ and $n / 4$ Hopf-Galois structures of type $D_{n / 2} \times C_{2}$ if $n \equiv 0(\bmod 4)$; and $n / 2$ Hopf-Galois structures of type $D_{n}$ if $n \equiv 2(\bmod 4)($ since $\left.D_{n / 2} \times C_{2} \cong D_{n}\right)$.

Case $4\left(\psi(r)=\psi(s)=r^{i} s, 0 \leq i \leq(n / 2)-1\right)$. (Note that $\psi(r)=r^{i} s, \psi(s)=$ $r^{j} s, 0 \leq i, j \leq(n / 2)-1$ is abelian if and only if $i=j$.) In this case, $r^{i} s$ is a fixed 
point if and only if $i$ is even. Thus, for $i$ odd we get a Hopf-Galois structure of type $D_{n}$. On the other hand, if $i$ is even then $G_{0}=\left\langle r^{2}, r s\right\rangle \cong D_{n / 2}, G_{1}=\left\langle r^{i} s\right\rangle$, and the Hopf-Galois structure is of type $D_{n / 2} \times C_{2}$.

The Hopf-Galois structure types are summarized in the following table.

\begin{tabular}{c|c|c|c|c|c}
$n$ & \# type $D_{n}$ & \# type $D_{n / 2} \times C_{2}$ & \# type $C_{n} \times C_{2}$ & \# abelian maps & \# HGS \\
\hline$n=4$ & 3 & 2 & 2 & 7 & 10 \\
\hline $2 \mid n, n>4$ & $1+n / 2$ & $n / 2$ & $n / 2$ & $1+3 n / 2$ & $2+5 n / 2$ \\
\hline $2 \nmid n$ & 1 & N/A & $n$ & $1+n$ & $2+n$
\end{tabular}

The rightmost column counts the total number of regular, $G$-stable subgroups found using abelian maps directly or through their opposite structures. The figures in this column are obtained by doubling the total number of abelian maps giving structures of nonabelian type and adding this sum to the total number of abelian maps giving structures of abelian type. We justify this below. The fact that $D_{n / 2} \times C_{2}$ is abelian if and only if $n=4$ accounts for the exceptional behavior for $n=4$ : note that the number of abelian maps for $n=4$ follows the more general formulas for the $2 \mid n, n>4$ case.

Our method of choosing $\psi$, where we avoid selecting nontrivial central elements for $\psi(r)$ and $\psi(s)$, ensures that the resulting regular, $G$-stable subgroups (and hence Hopf Galois structures) will all be distinct. It follows that the regular, $G$-stable subgroups arising from the opposite structures are necessarily distinct as well.

Finally, a nonabelian regular, $G$-stable subgroup arising from the opposite structure corresponding to an abelian map will never be realizable as a structure coming directly from an abelian map, i.e., there is no pair $\psi, \psi^{\prime}$ of abelian maps on $G$ with $g \psi^{\prime}\left(g^{-1}\right) h \psi^{\prime}(g)=h \psi\left(h^{-1}\right) g \psi(h)$ for all $g, h \in G$. To see this, let $h \in \operatorname{ker} \psi$ with $h \notin Z(G)$ : one can pick either $h=s$ or $h=r s$ in each of the cases above. Then $g \psi^{\prime}\left(g^{-1}\right) h \psi^{\prime}(g)=h g$ for all $g \in G$, i.e., $h=\left(g \psi^{\prime}\left(g^{-1}\right)\right) h\left(g \psi^{\prime}\left(g^{-1}\right)\right)^{-1}$. If we let $g=r^{2}$ then $g \psi\left(g^{-1}\right)=r^{2} \psi\left(r^{2}\right)=r^{2}$. Since $r^{2} s r^{2}=r^{4} s$ and $r^{2}(r s) r^{2}=r^{4}(r s)$ we see that $g \psi^{\prime}\left(g^{-1}\right) h \psi^{\prime}(g) \neq h g$ for $n \neq 4$. In the case $n=4$ all nonabelian regular, $G$-stable subgroups arise from abelian maps which are fixed point free; as $\left\{g \psi\left(g^{-1}\right): g \in G\right\}=G$ if and only if $\psi$ is fixed point free it would follow that $h \in Z(G)$, a contradiction. Thus the Hopf Galois structures constructed above are all distinct.

The number of structures of type $D_{n}\left(\right.$ or $D_{n / 2} \times C_{2}$ if $\left.n \equiv 2(\bmod 4)\right)$ arising from fixed point free abelian maps agrees with the results found in [7, §5] before opposites are considered.

\section{ACKNOWLEDGMENT}

The author would like to thank the referee for providing the useful suggestions provided during the revision of this paper.

\section{REFERENCES}

[1] David Bachiller, Counterexample to a conjecture about braces, J. Algebra 453 (2016), 160176, DOI 10.1016/j.jalgebra.2016.01.011. MR3465351

[2] Nigel P. Byott, Monogenic Hopf orders and associated orders of valuation rings, J. Algebra 275 (2004), no. 2, 575-599, DOI 10.1016/j.jalgebra.2003.07.003. MR2052627

[3] A. Caranti, Quasi-inverse endomorphisms, J. Group Theory 16 (2013), no. 5, 779-792, DOI 10.1515/jgt-2013-0012. MR3101012 
[4] Scott Carnahan and Lindsay Childs, Counting Hopf Galois structures on non-abelian Galois field extensions, J. Algebra 218 (1999), no. 1, 81-92, DOI 10.1006/jabr.1999.7861. MR.1704676

[5] Lindsay N. Childs, Bi-skew braces and Hopf Galois structures, New York J. Math. 25 (2019), 574-588. MR.3982254

[6] Lindsay N. Childs, Taming wild extensions: Hopf algebras and local Galois module theory, Mathematical Surveys and Monographs, vol. 80, American Mathematical Society, Providence, RI, 2000, DOI 10.1090/surv/080. MR.1767499

[7] Lindsay N. Childs, Fixed-point free endomorphisms and Hopf Galois structures, Proc. Amer. Math. Soc. 141 (2013), no. 4, 1255-1265, DOI 10.1090/S0002-9939-2012-11418-2. MR 3008873

[8] Teresa Crespo, Anna Rio, and Montserrat Vela, Induced Hopf Galois structures, J. Algebra 457 (2016), 312-322, DOI 10.1016/j.jalgebra.2016.03.012. MR.3490084

[9] Teresa Crespo, Anna Rio, and Montserrat Vela, Hopf Galois structures on symmetric and alternating extensions, New York J. Math. 24 (2018), 451-457. MR3855635

[10] Cornelius Greither and Bodo Pareigis, Hopf Galois theory for separable field extensions, J. Algebra 106 (1987), no. 1, 239-258, DOI 10.1016/0021-8693(87)90029-9. MR878476

[11] L. Guarnieri and L. Vendramin, Skew braces and the Yang-Baxter equation, Math. Comp. 86 (2017), no. 307, 2519-2534, DOI 10.1090/mcom/3161. MR.3647970

[12] Alan Koch, Timothy Kohl, Paul J. Truman, and Robert Underwood, Isomorphism problems for Hopf-Galois structures on separable field extensions, J. Pure Appl. Algebra 223 (2019), no. 5, 2230-2245, DOI 10.1016/j.jpaa.2018.07.014. MR.3906546

[13] Alan Koch, Timothy Kohl, Paul J. Truman, and Robert Underwood, Normality and short exact sequences of Hopf-Galois structures, Comm. Algebra 47 (2019), no. 5, 2086-2101, DOI 10.1080/00927872.2018.1529237. MR3977722

[14] Alan Koch, Laura Stordy, and Paul J. Truman, Abelian fixed point free endomorphisms and the Yang-Baxter equation, New York J. Math. 26 (2020), 1473-1492, DOI 10.3150/19bej1168. MR 4184834

[15] Alan Koch and Paul J. Truman, Opposite skew left braces and applications, J. Algebra 546 (2020), 218-235, DOI 10.1016/j.jalgebra.2019.10.033. MR4033084

[16] Alan Koch and Paul J. Truman, Skew left braces and isomorphism problems for Hopf-Galois structures on Galois extensions, arXiv:2005.05809, 2020.

[17] Wolfgang Rump, Braces, radical rings, and the quantum Yang-Baxter equation, J. Algebra 307 (2007), no. 1, 153-170, DOI 10.1016/j.jalgebra.2006.03.040. MR2278047

[18] Wolfgang Rump, A covering theory for non-involutive set-theoretic solutions to the YangBaxter equation, J. Algebra 520 (2019), 136-170, DOI 10.1016/j.jalgebra.2018.11.007. MR3881192

[19] Agata Smoktunowicz and Leandro Vendramin, On skew braces (with an appendix by $N$. Byott and L. Vendramin), J. Comb. Algebra 2 (2018), no. 1, 47-86, DOI 10.4171/JCA/2-13. MR 3763907

[20] Paul J. Truman, Canonical nonclassical Hopf-Galois module structure of nonabelian Galois extensions, Comm. Algebra 44 (2016), no. 3, 1119-1130, DOI 10.1080/00927872.2014.999930. MR3463133

Department of Mathematics, Agnes Scott College, 141 E. College Avenue, Decatur, GeOrgia 30030

Email address: akoch@agnesscott.edu 